\title{
Coal/biomass co-combustion investigation by thermogravimetric analysis
}

\author{
Akarasingh Bampenrat ${ }^{1, *}$, Hussanai Sukkathanyawat ${ }^{1}$, and Thidaporn Seangwattana ${ }^{1}$ \\ ${ }^{1}$ Faculty of Science Energy and Environment, King Mongkut's University of Technology North Bangkok (Rayong Campus), Rayong, \\ Thailand, 21120
}

\begin{abstract}
In this study, the thermal characteristics and kinetic parameters of coal/biomass blended fuels (75:25, 50:50 and 25:75 wt.\%/wt.\%) were investigated by using the thermogravimetric technique under atmospheric air. Three types of agricultural waste biomass including cassava root, palm kernel shell and rice husk were used as raw materials. The experiments were performed under different temperatures, ranging from 313-973 $\mathrm{K}$ with the heating rate of $5,10,20$ and $40 \mathrm{~K} / \mathrm{min}$. The results show that the thermal decomposition of biomass exhibit three-four stages including moisture and some light volatile removal stage (up to $463 \mathrm{~K}$ ), volatile oxidation stage (423-663 K), char combustion stage (663-823 K) and inorganic oxidation stage (803-953 K). Lignite on the other hand exhibits only two main peaks during the entire combustion process, corresponding to the moisture removal (up to $433 \mathrm{~K}$ ) and the decomposition/oxidation (433-833 K), respectively. In addition, it was also found that the blending of biomass residues improved the ignition temperature of the blended fuels, indicating an improvement of devolatilization of coal. Kinetic studies show that the average apparent activation energies of the co-combustion of coal/cassava root, coal/palm kernel shell and coal/rice husk calculated from the Kissinger-Akahira-Sunose method are reported at ca. $105.25,179.66$ and $121.84 \mathrm{~kJ} / \mathrm{mol}$, respectively.
\end{abstract}

Keyword. Biomass, Coal, Co-combustion, Thermogravimetric analysis

\section{Introduction}

In Thailand, the domestic resource potentials of major biomass residues, namely cassava root, palm kernel shell and rice husk, are about $6.1,0.5$ and 8.2 million tons per year, respectively, according to the Department of Alternative Energy Development and Efficiency, Ministry of Energy. Most of these agricultural and agroindustrial residues are utilized for power generation. However, the electricity produced in the country is still based on fossil fuel power plants. In order to reduce the consumption of fossil fuels, the co-combustion or cofiring technology is one of the most promising options for applications with renewable fuels. Co-combustion of solid fossil fuel with biomass benefits in terms of cost and emission reductions. In addition, co-combustion of biomass with coal in conventional coal-fired boilers can provide a reasonability attractive option for utilization of biomass for power generation in which using the infrastructure that is associated with the existing fossil fuel-based power system and requires some capital investment [1].

Thermo-analytical methods, such as thermogravimetric analysis (TGA or TG), differential thermogravimetry (DTG), differential thermal analysis (DTA) and differential scanning calorimetry (DSC), have been used to evaluate the measurements of mass and energy variations which cover a wide range of applications in research and development. For example, the DTG thermograms were employed to determine the decomposition temperatures of biopolymer templates for photocatalyst preparation [2], the TGA/DTA thermograms were used to obtain the thermal stability information of antibacterial filler prepared from leather industrial waste [3], the TGA and DSC data were applied to analyze the reaction areas, characteristic temperatures and heat flow rates of pellets derived from blends of animal manure and lignocellulosic biomass [4], and so on. Among these techniques, TGA, along with DTG, is one of the most approved techniques to provide a rapid assessment of the thermal characteristics during the combustion of solid fuels. Besides decomposition behavior, quantitative methods can be applied to TGA profiles in order to obtain kinetic parameters as reported in the cases of co-combustion of coal with pine sawdust [5], coconut fibers and eucalyptus leaves [6], lignocellulosic and animal wastes [7] and wood waste and rice straw [8], etc. Thermal decomposition characteristics and kinetic parameters of biomass, coal, as well as their blends provide pivotal information to evaluate energy potential of renewable feedstocks. Up to date, numerous TGA experiments in the field of biomass/coal co-combustion have been studied continually. For instance, F. Guo et al. [9] investigated the co-combustion of biomass pellets and the two kinds of coal using a thermogravimetric analyzer. They found

* Corresponding author: akarasingh.b@ sciee.kmutnb.ac.th 
that by increasing biomass pellet ratio, the maximum combustion rate and combustion index increased, while the burnout temperature decreased, indicating that the combustion performance of coal can be improved. S. Qi et al. [10] evaluated the ignition and combustion characteristics of single particles of two biomass residues (corn straw and wheat straw) and three coals (lignite, bituminous and anthacite coals) in $\mathrm{N}_{2} / \mathrm{O}_{2}$ and $\mathrm{CO}_{2} / \mathrm{O}_{2}$ environments. They reported that the biomass and lignite particles ignited homogeneously, while the bituminous coal and anthracite particles ignited heterogeneously. Moreover, the biomass particles ignited earlier than the coal particles. For the three coals, the ignition delay time decreased almost linearly with the increase in their volatiles contents.

Due to the combustion characteristics and reaction of biomass, composed of hemicellulose, cellulose and lignin, are quite different. Therefore, the purpose of this research is to investigate the combustion characteristics of the three abundant biomass wastes in Thailand, i.e., cassava root, palm kernel shell and rice husk, as well as the influence of these residues when co-combusted with lignite coal. The experimental results of the cocombustion between lignite coal and such three biomass wastes discussed in this article would be helpful to promote the utilization of coal/biomass co-combustion for energy production and also to reduce the coal consumption (partially substitute for coal) in any coalbased thermal power plants in Thailand.

\section{Materials and methods}

\subsection{Sample preparation and characterisation}

Lignite and palm kernel shell samples were provided by an industry, while cassava root and rice husk are obtained from the local agricultural areas of Mabkha City, Rayong Province, Thailand. The biomass samples were washed with water, cut into small pieces and naturally dried for 3-5 days. Using a grinding machine, the sample was ground and was sieved to collect the fraction with particle size smaller than $250 \mu \mathrm{m}$ for further study. The individual raw materials were named C100 (lignite coal), CR100 (cassava root), PKS100 (palm kernel shell) and RH100 (rice husk). Different mixtures of lignite coal and biomass residues were prepared by physical mixing and were denoted by CXCRY, CXPKSY and CXRHY, where $\mathrm{X}=75,50$ and $25 ; \mathrm{Y}=25,50$ and $75 \mathrm{wt} . \% / \mathrm{wt} . \%$ for coal/cassava root, coal/palm kernel shell and coal/rick husk blends, respectively. The proximate analyses including moisture content $\mathrm{M}$ ), volatile matter (VM), and ash content (A) were determined according to ASTM E870-82, while the fixed carbon (FC) was calculated by subtracting the percentages of moisture content, volatile matter and ash content from $100 \%$. In addition, the calorific value or higher heating value (HHV) of samples was calculated by the data obtained from the oxygen bomb calorimeter (Parr 1341).

\subsection{Thermogravimetric experiments}

The combustion behavior of solid fuel samples was carried out with TA Instrument Q50 analyser under an air atmosphere. Prior to the TGA experiments, the fine sample powder, ca. 5-10 mg, was placed in a small platinum crucible and thermally purged under a nitrogen stream at $313 \mathrm{~K}$ for $10 \mathrm{~min}$. Thermogravimetry (TG) and derivative thermogravimetric (DTG) profiles were attained by heating the sample from 313 to $973 \mathrm{~K}$ using an air flow of $60 \mathrm{ml} / \mathrm{min}$, with the heating rate of 20 $\mathrm{K} / \mathrm{min}$. Analysis of each sample was repeated at least twice and the repeatability of experiments was more than $99 \%$. In an attempt to obtained the kinetic parameters of coal/biomass co-combustion, the heating rates of 5, 10, 20 and $40 \mathrm{~K} / \mathrm{min}$ were used for kinetic analysis.

\subsection{Kinetic procedure}

The reaction rate $(r)$ of co-combustion of coal-biomass blends may be expressed by the following equation:

$$
r=\frac{d \alpha}{d t}=k(T) f(\alpha)=A e^{\left(-E_{a} / R T\right)} f(\alpha)
$$

where $k(T)$ is the reaction rate constant, $T$ is the absolute temperature, $f(\alpha)$ is the function of reaction mechanism, $A$ is the frequency factor, $E_{a}$ is the activation energy, $R$ is the universal gas constant and $\alpha$ is the fractional change in solid mass due to thermal decomposition at any time $t$ or conversion, given by the equation:

$$
\alpha=\frac{m_{0}-m_{t}}{m_{0}-m_{f}}
$$

where $m_{0}, m_{t}$ and $m_{f}$ are the initial mass, the instantaneous mass and the final mass of the solid sample, respectively. Under constant heating rate, $\beta=d T / d t$, and integrating both sides of equation (1) give:

$$
\begin{aligned}
g(\alpha)=\int_{0}^{\alpha} \frac{d \alpha}{f(\alpha)}=\frac{A}{\beta} \int_{T_{0}}^{T} e^{\left(-E_{a} / R T\right)} d T & =\frac{A E_{a}}{\beta R} p\left(\frac{E_{a}}{R T}\right) \\
& =\frac{A E_{a}}{\beta R} p(u)
\end{aligned}
$$

where $g(\alpha)$ is the integral form of the conversion dependence function and $p(u)$ is the temperature integral function which is no analytical solution.

The most common soluion used to determine the kinetic parameters is the isoconversional method or model-free method. Isoconversional method has been proven effective particularly for kinetic analysis and has recently been strongly recommended by the Kinetics Committee of the International Confederation for Thermal Analysis and Calorimetry (ICTAC) [11]. By 
applying the Kissinger-Akahira-Sunose (KAS) method, one of the isoconversional method, in Equation (3), the KAS equation can be expressed as:

$$
\ln \left(\frac{\beta}{T^{2}}\right)=\ln \left[\frac{A R}{E_{a} g(\alpha)}\right]-\frac{E_{a}}{R T}
$$

Ploting $\ln \left(\beta / T^{2}\right)$ against $(1 / T)$, the activation energy was determined from the slope of the resulting straight line. In order to find the frequency factor, the general thermal decomposition reaction represented by $f(\alpha)=(1-\alpha)$ was used in this study.

\section{Results and discussion}

\subsection{Physical properties of sample}

The basic characteristics, including M, VM, FC, A and calorific value, of lignite and biomass wastes are presented in Table 1. It was observed that $\mathrm{C} 100$ is dominated by fixed carbon content (32.04 wt.\%), whereas the biomass wastes, CR100, PKS100 and RH100, are prevailed by volatile matter (54.96-75.25 wt.\%) and relatively low ash content except rice husk. High amount of ash in the case of RH100 can be explained by high silica content [12]. Considering the key elements involved in the initiation of ignition, some research studies [13, 14] reveal that biomass contain higher volatile matter and volatile fuel ratio $[\mathrm{VM} /(\mathrm{VM}+\mathrm{FC})]$ than coal. In this work, the values of volatile fuel ratio for biomass residues are in the range of 0.74-0.89 higher than that of lignite coal (0.63). This indicates that biomass residues have a higher reactivity and are easier to achieve ignition. Therefore, it can be expected that the blending of biomass waste and coal would enhance the ignition property of fuel.

Table 1. Characteristics of coal and biomass wastes.

\begin{tabular}{|c|c|c|c|c|c|}
\hline \multirow{2}{*}{ Sample } & \multicolumn{3}{|c|}{ Proximate analysis (wt.\%) } & \multirow{2}{*}{ HHV } \\
\cline { 2 - 5 } & M & VM & FC & A & (MJ/kg) \\
\hline C100 & 8.97 & 53.54 & 32.04 & 5.45 & 23.77 \\
\hline CR100 & 9.05 & 75.07 & 8.94 & 6.94 & 16.94 \\
\hline PKS100 & 7.62 & 72.25 & 13.52 & 6.61 & 21.25 \\
\hline RH100 & 7.98 & 54.96 & 19.29 & 17.78 & 14.29 \\
\hline
\end{tabular}

Based on data obtained from the oxygen bomb calorimeter, the higher heating value of $\mathrm{C} 100$ is 23.77 $\mathrm{MJ} / \mathrm{kg}$, while the calorific values of biomass wastes are 16.94, 21.25, and 14.29 MJ/kg for CR100, PKS100, and RH100, respectively, which are located at the normal $\mathrm{HHV}$ range (i.e., 14-20 MJ/kg) of biomass [15].

\subsection{Combustion behavior of individual fuels}

Figure 1 and Table 2 show the typical TG curve, DTG curve and decomposition temperature intervals of lignite coal and biomass wastes decomposed under atmospheric air. According to the derivative weight loss, agricultural and agro-industrial residues exhibit 3-4 degradation stages reflecting the existence of structures of different reactivity. Whereas lignite coal shows only two characteristic peaks indicating more homogeneous material.
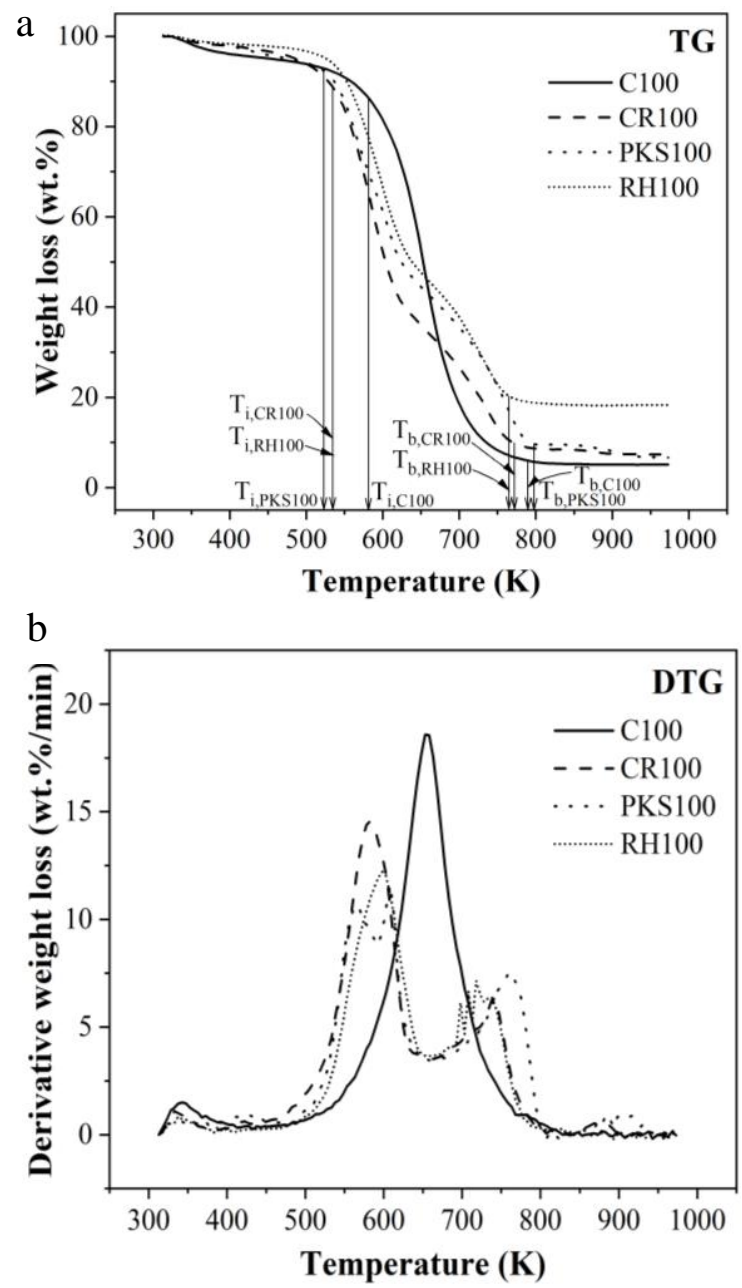

Fig. 1. a) TG and b) DTG profiles of coal and biomass wastes combustions with the heating rate of $20 \mathrm{~K} / \mathrm{min}$.

Table 2. Temperature intervals for different mass loss regions.

\begin{tabular}{|c|c|c|c|c|}
\hline \multirow{2}{*}{ Sample } & \multicolumn{4}{|c|}{ Temperature range (K) } \\
\cline { 2 - 5 } & Stage 1 & Stage 2 & Stage 3 & Stage 4 \\
\hline C100 & $300-433$ & $433-833$ & - & - \\
\hline CR100 & $300-433$ & $433-663$ & $663-803$ & $803-933$ \\
\hline PKS100 & $300-463$ & $463-663$ & $663-823$ & $823-953$ \\
\hline RH100 & $300-423$ & $423-663$ & $663-823$ & - \\
\hline
\end{tabular}


The mass loss during the first stage of both the lignite coal and biomass wastes are normally due to the evaporation of free moisture, inherent moisture as well as low-boiling volatile release occurred at the temperature up to $463 \mathrm{~K}$. For C100 sample, the main peak occurs in the second stage between 433 and $833 \mathrm{~K}$ with the maximum weight loss rate $\left(R_{\max }\right)$ of 18.67 wt. $\% / \mathrm{min}$, which was caused by the simultaneous combustion of volatile and solid carbon. In contrary to coal, biomass residues, CR100, PKS100 and RH100, exhibit 2-3 more decomposition stages after evaporation stage with the maximum weight loss rates between 11.29 and 14.57 wt.\%/min. Stage 2 occurs between 423 and $663 \mathrm{~K}$ and stage 3 occurs in the temperature range of 663-823 K, which are ascribed as the volatilization of three main constituents (hemicellulose, cellulose and lignin) of biomass and the combustion of char residue, respectively. Nevertheless, some researchers $[9,16]$ claimed that the mass loss during the second stage is attributed to the total decomposition of hemicellulose and cellulose and the partial decomposition of lignin and during the third stage by the decomposition of remaining lignin and combustion of char residue. Another thermal decomposition stage (stage 4) in the temperature range of 803-953 K can be observed in the cases of CR100 and PKS100, which is attributed to the inorganic material decomposition [14]. It is noteworthy that the maximum weight loss rate of $\mathrm{C} 100$ is highest. Moreover, all biomass wastes lost their mass rapidly in the temperature below $650 \mathrm{~K}$, while lignite coal sample decomposed rapidly at the temperature above $650 \mathrm{~K}$. These circumstances imply that the incorporation of biomass wastes into lignite coal might not be totally benefit in terms of combustion performance.

Ignition temperature $\left(T_{i}\right)$ and burnout temperature $\left(T_{b}\right)$ are important properties of solid fuels for their industrial application. A number of methods have been developed to figure out the ignition and burnout temperatures of fuels, i.e., intersection method, derivation method, and conversion method. In this study, the ignition and burnout temperatures were determined following the intersection method as described by $\mathrm{Lu}$ and Chen [17]. Figure 2 illustrates the schematic of the ignition and burnout temperature determinations for CR100 sample by using the intersection method. Two points, A and B, on a TG curve are first identified. Point $\mathrm{A}$ is the point at which a vertical line from the highest value in the DTG curve crosses the TG curve. Point $B$ is the point at which devolatilization begins (about 378-383 $\mathrm{K})$. A tangent at point $\mathrm{A}$ on the TG curve and the horizontal line through point $\mathrm{B}$ are drawn. The corresponding temperature at the intersection of two lines is identified as the ignition temperature. In order to determine the burnout temperature of fuel sample, points $\mathrm{C}$ and $\mathrm{D}$ are located. Point $\mathrm{C}$ is the position on the TG curve at which a vertical line from the second peak of the DTG curve crosses the TG curve, while point D is the location at which the TG curve becomes steady. A temperature corresponding to the intersection of the tangent on the TG curve at $\mathrm{C}$ and the horizontal line through $\mathrm{D}$ is defined as the burnout temperature.

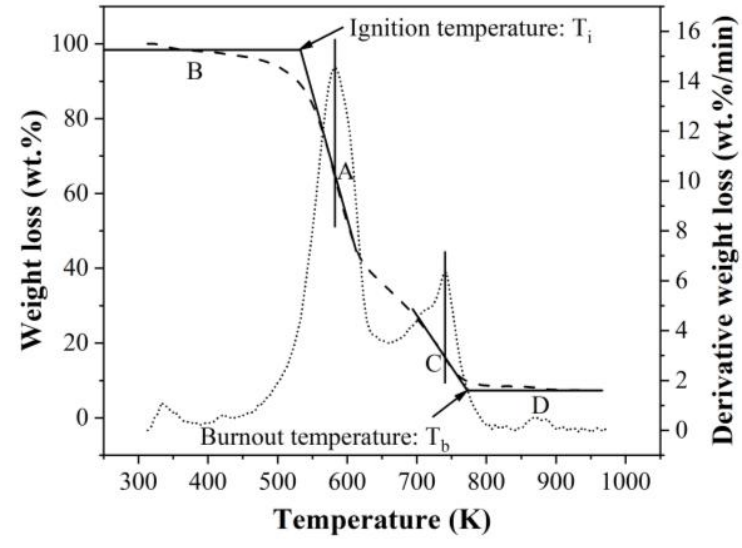

Fig 2. Schematic of the ignition and burnout temperature determinations.

The result of combustion characteristic parameters of individual sample is given in Table 3 . It is noted that in the cases of cassava root and palm kernel shell the weight loss occurred above $800 \mathrm{~K}$, due to the inorganic substance decompositions, were not considered for burnout temperature determination. It was found that the ignition temperatures of biomass residues are lower by $45-57 \mathrm{~K}$ than that of lignite coal. This could be enhanced the ignition performance of blended fuels caused by the blending of biomass wastes into coal. In terms of the burnout temperature, however, palm kernel shell shows higher value than lignite $(796 \mathrm{~K}>790 \mathrm{~K})$. This implies that some volatiles released during the early combustion of PSK100 inhibit the char or heavy volatile combustion and thereby caused a longer complete combustion. The total burnout of C100, CR100, PKS100 and RH100 are about $94.85,92.64,93.32$, and 81.70 wt.\%, respectively.

Table 3. Characteristic parameters of coal and biomass wastes.

\begin{tabular}{|c|c|c|c|c|}
\hline Sample & $\mathbf{T}_{\mathbf{i}}(\mathbf{K})$ & $\mathbf{T}_{\mathbf{b}}$ (K) & $\begin{array}{c}\mathbf{R}_{\max } \\
\text { (wt.\%/min) }\end{array}$ & $\begin{array}{c}\text { Total } \\
\text { burn out (wt.\%) }\end{array}$ \\
\hline C100 & 580 & 790 & 18.67 & 94.85 \\
\hline CR100 & 535 & 772 & 14.57 & 92.64 \\
\hline PKS100 & 523 & 796 & 11.29 & 93.32 \\
\hline RH100 & 535 & 764 & 12.16 & 81.70 \\
\hline
\end{tabular}

Note: $\mathrm{T}_{\mathrm{i}}$, ignition temperature; $\mathrm{T}_{\mathrm{b}}$, burnout temperature; $\mathrm{R}_{\max }$, maximum mass loss rate.

\subsection{Co-combustion of fuels}

The TG and DTG profiles of co-combustion of coal/biomass blended fuels with different percentages are shown in Figure 3-5. A similar trend can be seen for all biomass blended where the combustion of samples takes place with combined weight loss lied between that of the individual fuel. Similar phenomena were also found in the case of other blending samples $[13,14]$. With considering the example of coal/biomass cocombustion in present study, C50CR50 (equally 
C25CR75) decomposes rapidly and more reactive than $\mathrm{C} 100$ at the temperature below $660 \mathrm{~K}$. However, C75CR25 exhibits the most reactivity at the temperature above $660 \mathrm{~K}$ but it still lower than that of C100. In addition, the maximum weight loss rate of the combustion of coal component shifts to higher temperature (from $654 \mathrm{~K}$ to $678 \mathrm{~K}$ ) with increasing of biomass portions. For coal-palm kernel shell blended fuels, C50PKS50 losses its weight abruptly at the temperature below $650 \mathrm{~K}$, but at the temperature above $650 \mathrm{~K}$ C75PSK25 shows the most reactive for combustion. The maximum weight loss rate of the combustion of coal component, in addition, shifts to higher temperature from $655 \mathrm{~K}$ to $670 \mathrm{~K}$ with increasing of palm kernel shell portions. In the case of coal-rice husk blended fuels, C50RH50 exhibits the most reactivity at the temperature below $644 \mathrm{~K}$, while C75RH25 shows the most reactivity at the temperature above $644 \mathrm{~K}$. Moreover, the maximum combustion weight loss rate of coal component shifts to higher temperature from $656 \mathrm{~K}$ to $686 \mathrm{~K}$ with increasing of rice husk portions. These phenomena indicate that the combustion processes of the blended fuels are complex, which could be attributed to the interaction combustion behavior between biomass residues and lignite coal.

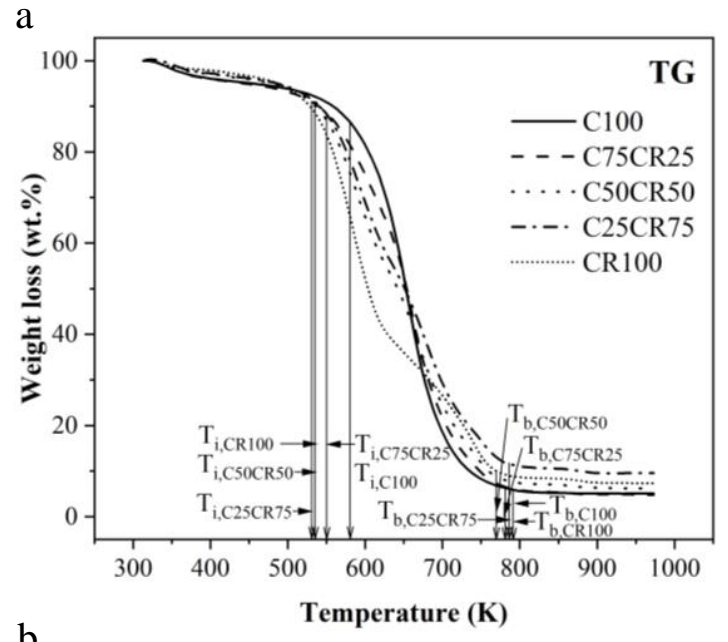

b

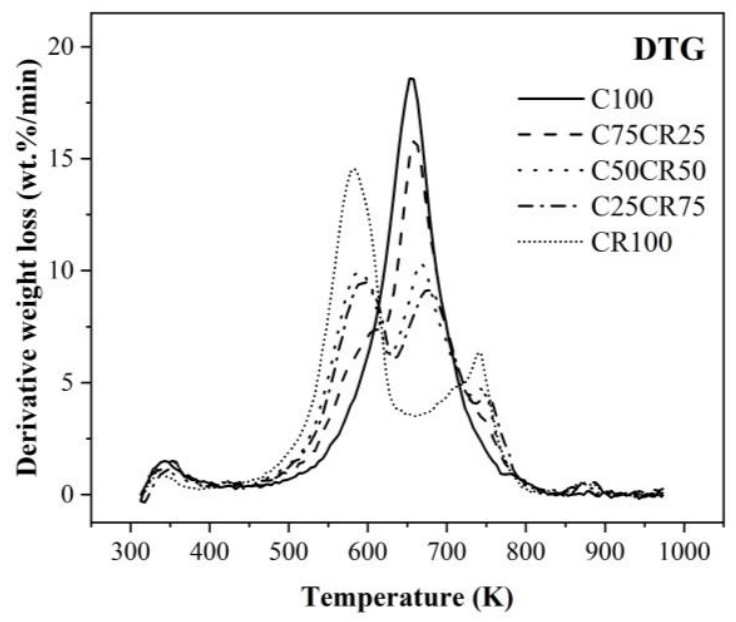

Fig. 3. a) TG and b) DTG profiles of coal/cassava root cocombustion.

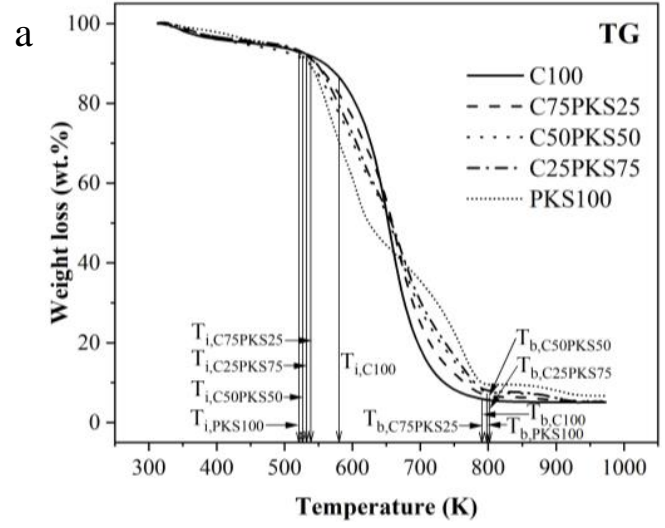

b

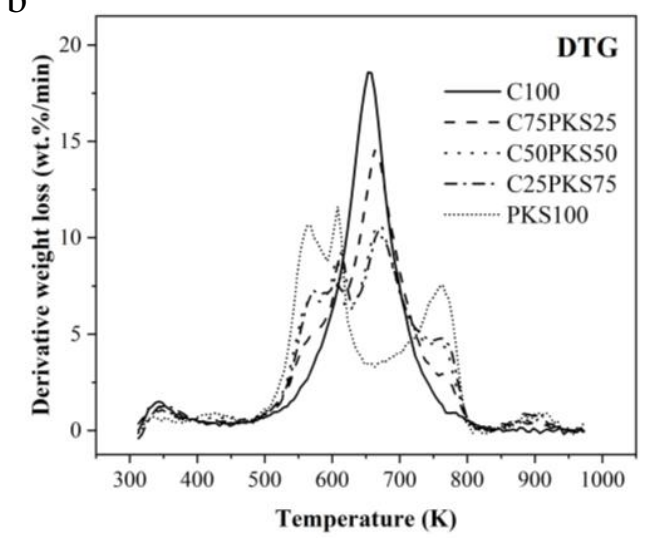

Fig. 4. a) TG and b) DTG profiles of coal/palm kernel shell cocombustion.

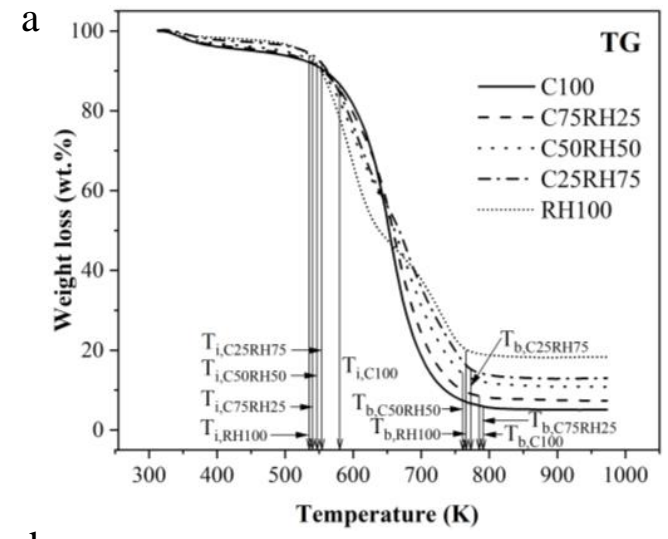

b

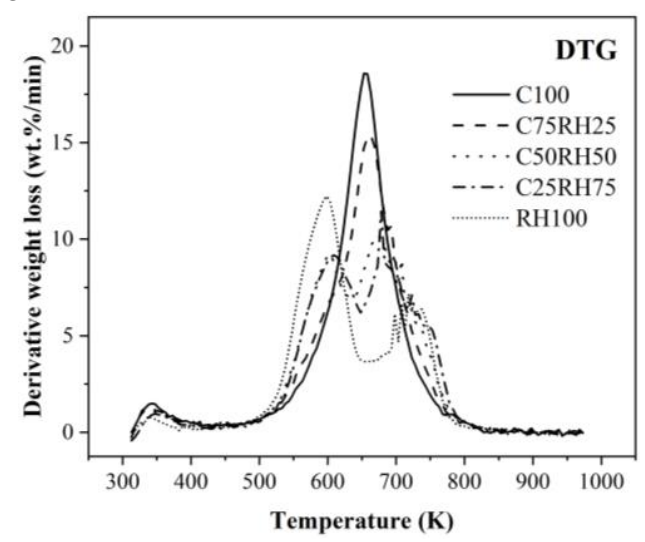

Fig. 5. a) TG and b) DTG profiles of coal/rice husk cocombustion. 
It can be briefly suggested as: (i) biomass residues have high content of volatile matters which easily decompose and ignite. Therefore, they could combust rapidly and release the energy preheating coal at the low temperature region, e.g., $<660 \mathrm{~K}$ for $\mathrm{C} 25 \mathrm{CR} 75$. In addition, the volatile compounds released abundantly consume a great amount of oxygen and thus hindered the oxygen molecules to reach the lignite coal surface, resulting in a lower reactivity of coal decomposition. So, this stage is dominated by the volatilization and volatile matter combustion (ii) when the temperature is higher $(>660 \mathrm{~K})$, most of volatile compounds released in the early stage are combust completely, accompanied with coal has enough energy to ignite and combust, the rapidly mass loss due to coal combustion could be observed beyond $660 \mathrm{~K}$. At this stage the fixed carbon combustion of coal is dominated.

In order to evaluate the combustion performance of coal/biomass co-combustion, two parameters, i.e., ignition index $\left(D_{i}\right)$ and combustion index $\left(S_{i}\right)$, have been used for various fuel systems $[16,18-20]$. The ignition and combustion index can be determined by the following equations:

$$
\begin{aligned}
& D_{i}=\frac{R_{\max }}{t_{i} t_{m}} \\
& S_{i}=\frac{R_{\max } R_{\text {mean }}}{T_{i}^{2} T_{b}}
\end{aligned}
$$

where $R_{\text {max }}$ and $R_{\text {mean }}$ are maximum and average mass loss rates, respectively. $T_{i}$ and $T_{b}$ stand for the ignition and burnout temperatures, respectively. $t_{i}$ and $t_{m}$ are the ignition time and the time which corresponds to maximum mass loss rate, respectively.

The result of characteristic combustion parameters calculated from the thermal decomposition profiles is shown in Table 4. It was unexpectedly found that although biomass residues improve the ignition temperature of the blended fuels (528-553 K) compared to coal $(580 \mathrm{~K})$, the ignition index of all blended $(7.08 \times$ $\left.10^{-4}-12.28 \times 10^{-4}\right)$ are lower than that of $\mathrm{C} 100(12.81 \times$ $10^{-4}$ ). Furthermore, the combustion index of the blended fuels are also lower than pure lignite coal, i.e., $27.42 \times$ $10^{-8}-47.48 \times 10^{-8}$ are lower than that of $53.95 \times 10^{-8}$. This might be because the maximum weight loss rates of the blended fuels are lower than that of coal. In fact, it can be remarked that most of research works $[5,9,13-14$, 21-23] studied on the low-grade coals, which have the lower maximum combustion rate than the studied biomass and less thermal decomposition reactivity.

Although, both of ignition and combustion index of coal/biomass-blended fuels in present study are lower than lignite coal, the utilization of biomass wastes approximately 25 wt.\% instead of coal could be recommended in which the combustion performances are lower than coal in the range of $11.45-19.35 \%$. The application in equal parts, however, the combustion index would be diminished by almost $50 \%$.

\subsection{Kinetic analysis}

The TG and DTG profiles of the C75CR25, C75PKS25 and $\mathrm{C} 75 \mathrm{RH} 25$ samples at different heating rates are shown in Fig. 6-8. It can be seen that the TG curves shift to high temperatures without a significant change in their shapes while the heating rate increases, suggested that the entire combustion process slows down with the increase of heating rate. Additionally, the increasing in heating rate made the maximum mass loss rate shift toward higher values, suggesting an improvement of combustion intensity. Similar phenomena were also found in the cases of other biomass residues $[8,13,16]$. Figures 9-11 show the KAS plots for kinetic parameter calculations and Table 5 summarises the kinetic parameters for coal/biomass co-combustion. It was found that the activation energies of coal/biomass cocombustion are in the range of 91.92-113.20, 141.05230.60 and $98.42-129.63 \mathrm{~kJ} / \mathrm{mol}$, which the average values are about $105.25,179.66$ and $121.84 \mathrm{~kJ} / \mathrm{mol}$ for C75CR25, C75PKS25 and C75RH25, respectively. It should be noticed that the activation energy of C75PKS25 is larger than those of C75CR25 and C75RH25, suggesting more energy requirement for coal/palm kernel sell co-combustion system. The variation of activation energy with respect to conversion, furthermore, can be observed, implying the complex reactions took place during the coal/biomass cocombustion process. The values of calculated frequency factors also show in the wide range: $6.62 \times 10^{5}-$ $6.26 \times 10^{7}, 1.62 \times 10^{8}-6.49 \times 10^{16}, 4.13 \times 10^{6}-9.60 \times 10^{8} \mathrm{~s}^{-1}$, for C75CR25, C75PKS25 and C75RH25, respectively.

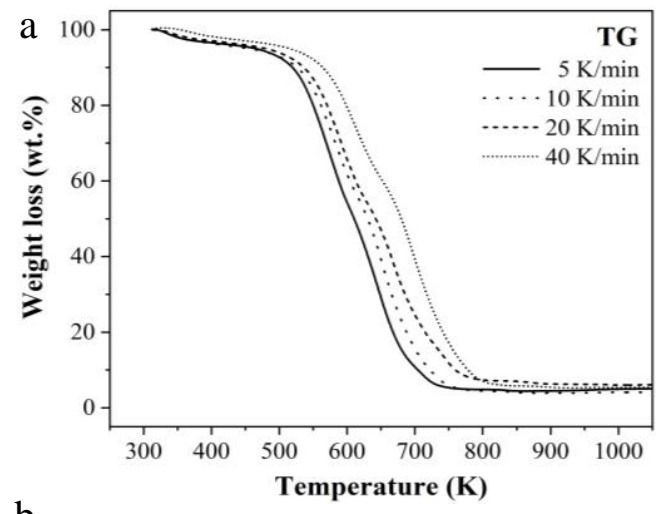

b

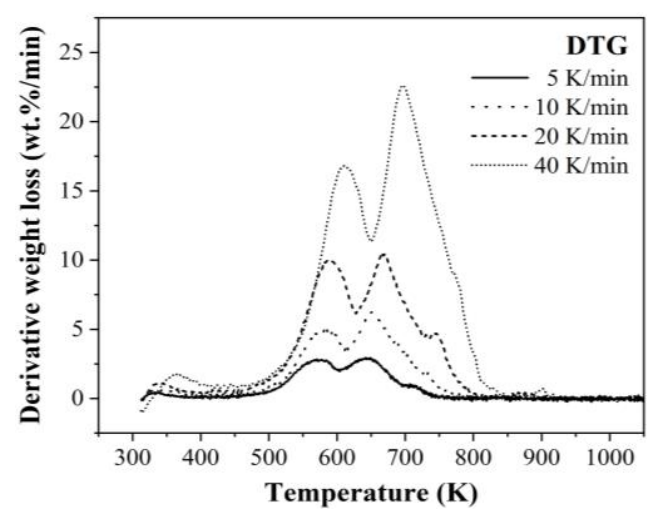

Fig. 6. a) TG and b) DTG profiles of C75CR25 co-combustion with different of heating rates. 


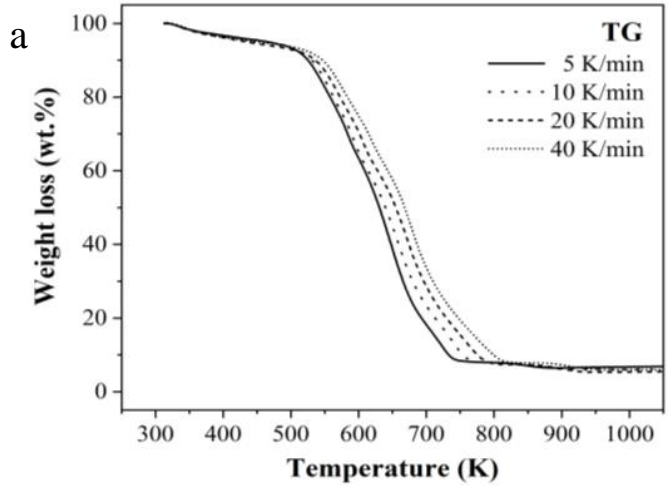

b

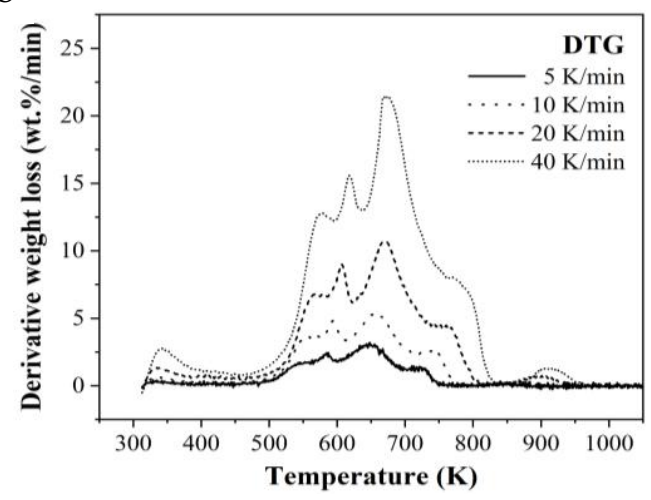

Fig. 7. a) TG and b) DTG profiles of C75PKS25 cocombustion with different of heating rates.

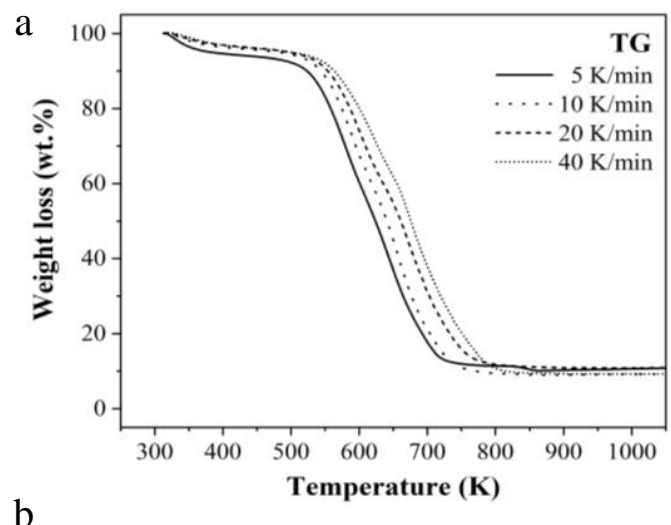

b

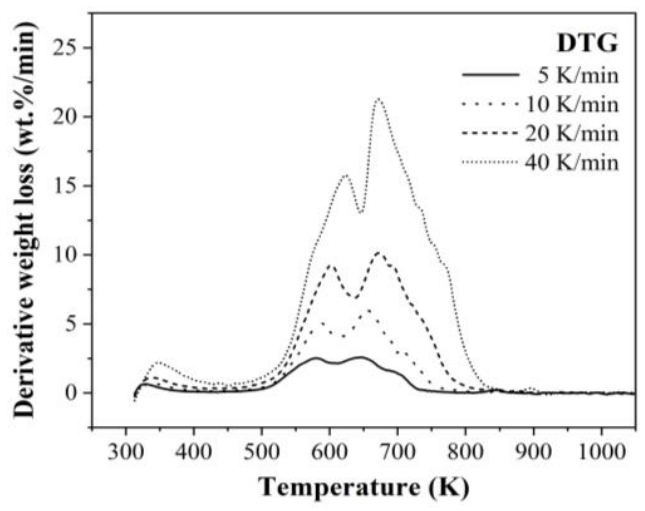

Fig. 8. a) TG and b) DTG profiles of C75RH25 co-combustion with different of heating rates.

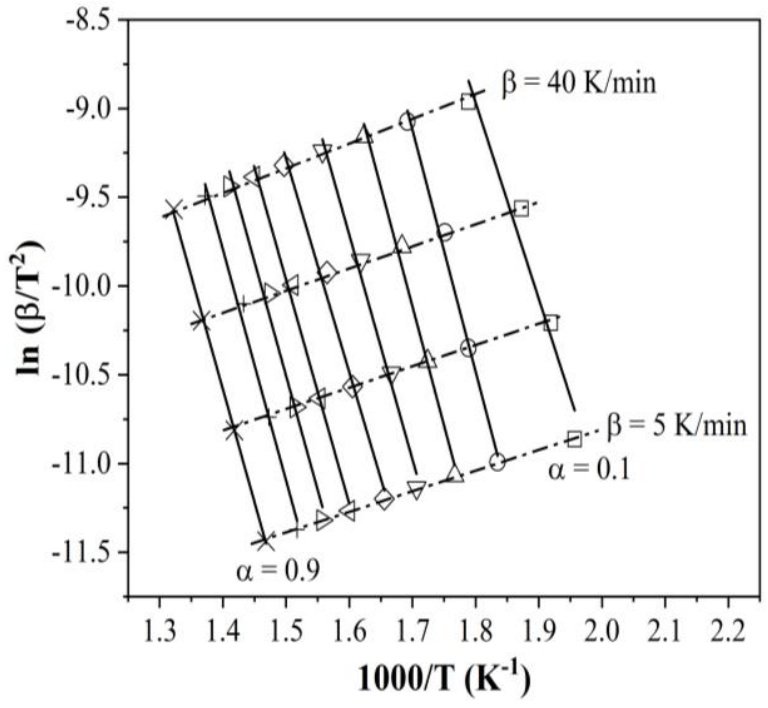

Fig. 9. KAS plot for C75CR25 co-combustion.

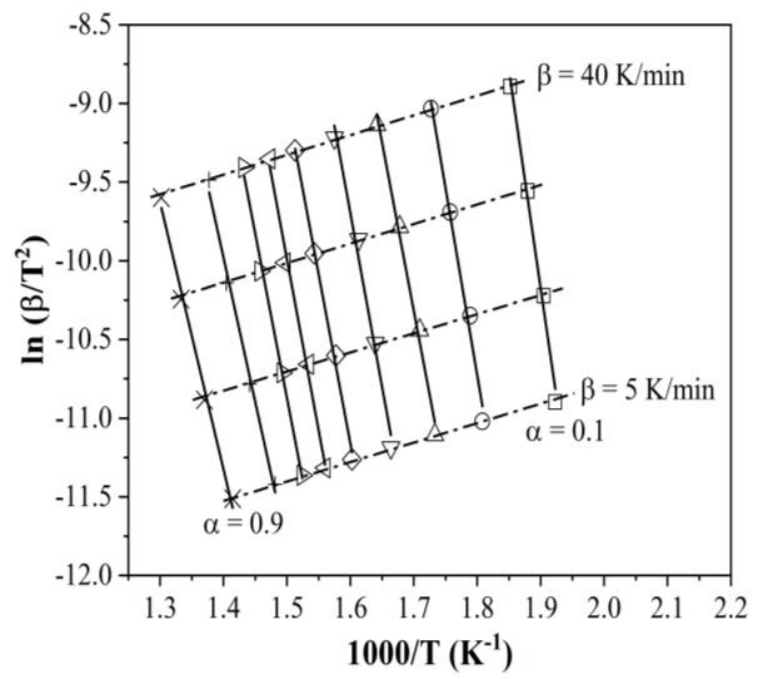

Fig. 10. KAS plot for C75PKS25 co-combustion.

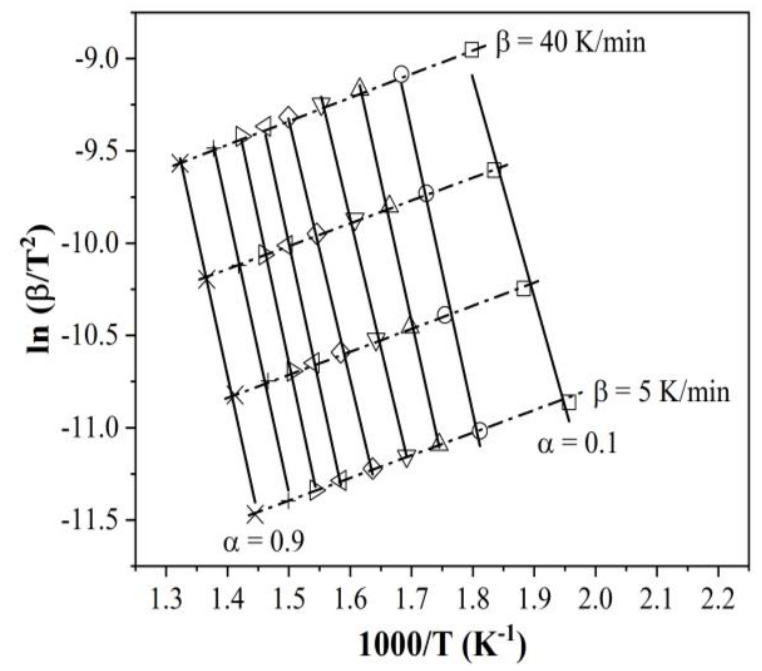

Fig. 11. KAS plot for C75RH25 co-combustion. 
Table 4. Characteristic parameters of co-combustion.

\begin{tabular}{|l|c|c|c|c|c|c|c|c|c|}
\hline \multicolumn{1}{|c|}{ Sample } & $\mathbf{T}_{\mathbf{i}}(\mathbf{K})$ & $\mathbf{T}_{\mathbf{b}}(\mathbf{K})$ & $\begin{array}{c}\mathbf{R}_{\mathbf{m a x}} \\
(\mathbf{w t .} \mathbf{\%} / \mathbf{m i n})\end{array}$ & $\mathbf{t}_{\mathbf{i}}(\mathbf{m i n})$ & $\mathbf{t}_{\mathbf{b}}(\mathbf{m i n})$ & $\mathbf{t}_{\mathbf{m}}(\mathbf{m i n})$ & $\begin{array}{c}\text { Total } \\
\text { Burnout (wt. } \mathbf{m})\end{array}$ & $\begin{array}{c}\mathbf{D}_{\mathbf{i}} \\
\left(\times \mathbf{1 0}^{-4}\right)\end{array}$ & $\begin{array}{c}\mathbf{S}_{\mathbf{i}} \\
\left(\times \mathbf{1 0}^{-8}\right)\end{array}$ \\
\hline C100 & 580 & 790 & 18.67 & 13.84 & 24.35 & 17.55 & 94.85 & 12.81 & 53.95 \\
\hline C75CR25 & 550 & 780 & 15.81 & 12.31 & 23.83 & 17.75 & 95.04 & 12.06 & 47.78 \\
\hline C50CR50 & 536 & 769 & 10.58 & 11.55 & 24.55 & 18.05 & 93.75 & 8.46 & 30.41 \\
\hline C25CR75 & 532 & 781 & 9.484 & 11.43 & 23.88 & 14.35 & 90.16 & 9.64 & 27.42 \\
\hline CR100 & 535 & 790 & 14.57 & 11.55 & 23.42 & 13.95 & 92.64 & 15.07 & 43.91 \\
\hline C75PKS25 & 536 & 789 & 14.81 & 12.03 & 24.67 & 18.45 & 94.66 & 11.12 & 43.51 \\
\hline C50PKS50 & 528 & 798 & 10.75 & 11.23 & 24.78 & 18.35 & 94.41 & 8.69 & 29.81 \\
\hline C25PKS75 & 530 & 801 & 10.42 & 11.35 & 24.91 & 18.15 & 94.74 & 8.43 & 28.90 \\
\hline PKS100 & 523 & 796 & 11.29 & 10.87 & 24.54 & 15.15 & 93.32 & 11.43 & 31.42 \\
\hline C75RH25 & 540 & 788 & 11.83 & 11.83 & 24.21 & 17.80 & 92.39 & 12.28 & 45.46 \\
\hline C50RH50 & 549 & 762 & 12.29 & 12.29 & 22.93 & 18.45 & 88.96 & 7.47 & 31.98 \\
\hline C25RH75 & 553 & 773 & 12.52 & 12.52 & 23.49 & 18.75 & 86.80 & 7.08 & 29.49 \\
\hline RH100 & 535 & 764 & 11.65 & 11.65 & 23.10 & 14.75 & 81.70 & 11.79 & 35.76 \\
\hline
\end{tabular}

Note: $\mathrm{T}_{\mathrm{i}}$, ignition temperature; $\mathrm{T}_{\mathrm{b}}$, burnout temperature; $\mathrm{R}_{\max }$, maximum mass loss rate; $\mathrm{t}_{\mathrm{i}}$, time at $\mathrm{T}_{\mathrm{i}} ; \mathrm{t}_{\mathrm{b}}$, time $\mathrm{at} \mathrm{T}_{\mathrm{b}} ; \mathrm{t}_{\mathrm{m}}$, time at $\mathrm{R}_{\max } ; \mathrm{D}_{\mathrm{i}}$, ignition index; $\mathrm{S}_{\mathrm{i}}$, combustion index.

Table 5. Kinetic parameters for coal/bio mass co-combustion by KAS method.

\begin{tabular}{|c|c|c|c|c|c|c|}
\hline \multirow{2}{*}{$\alpha$} & \multicolumn{2}{|c}{ C75CR25 } & \multicolumn{2}{c|}{ C75PKS25 } & \multicolumn{2}{c|}{ C75RH25 } \\
\cline { 2 - 7 } & $\boldsymbol{E}_{a}(\mathbf{k J} / \mathbf{m o l})$ & $\boldsymbol{A}\left(\mathbf{s}^{-1}\right)$ & $\boldsymbol{E}_{a}(\mathbf{k J} / \mathbf{m o l})$ & $\boldsymbol{A}\left(\mathbf{s}^{-1}\right)$ & $\boldsymbol{E}_{a}(\mathrm{~kJ} / \mathbf{m o l})$ & $\boldsymbol{A}\left(\mathbf{s}^{-1}\right)$ \\
\hline 0.1 & 91.92 & $1.09 \times 10^{6}$ & 230.60 & $6.49 \times 10^{16}$ & 98.42 & $4.13 \times 10^{6}$ \\
\hline 0.2 & 113.20 & $6.26 \times 10^{7}$ & 198.01 & $8.04 \times 10^{15}$ & 127.30 & $9.60 \times 10^{8}$ \\
\hline 0.3 & 111.48 & $2.56 \times 10^{7}$ & 177.74 & $2.55 \times 10^{13}$ & 125.75 & $3.93 \times 10^{8}$ \\
\hline 0.4 & 105.19 & $4.04 \times 10^{6}$ & 183.66 & $2.59 \times 10^{13}$ & 116.33 & $3.26 \times 10^{7}$ \\
\hline 0.5 & 99.87 & $8.63 \times 10^{5}$ & 178.80 & $3.11 \times 10^{12}$ & 116.71 & $2.01 \times 10^{7}$ \\
\hline 0.6 & 104.90 & $1.50 \times 10^{6}$ & 180.81 & $2.24 \times 10^{12}$ & 128.49 & $1.25 \times 10^{8}$ \\
\hline 0.7 & 106.79 & $1.65 \times 10^{6}$ & 173.90 & $3.37 \times 10^{11}$ & 129.63 & $1.07 \times 10^{8}$ \\
\hline 0.8 & 108.20 & $1.59 \times 10^{6}$ & 152.40 & $3.18 \times 10^{9}$ & 127.20 & $4.47 \times 10^{7}$ \\
\hline 0.9 & 105.67 & $6.62 \times 10^{5}$ & 141.05 & $1.62 \times 10^{8}$ & 126.71 & $2.39 \times 10^{7}$ \\
\hline Average & 105.25 & & 179.66 & & 121.84 & \\
\hline
\end{tabular}

Note: $\mathrm{R}^{2}$ are between 0.96 and 0.99 . 


\section{Conclusions}

It can be concluded that lignite coal mainly decomposes only two stages, i.e., evaporation and the simultaneous volatile and char combustions. Biomass residues, namely cassava root, palm kernel shell and rice husk, decompose three to four stages which are corresponding to the evaporation, volatilization, volatile and char combustions and inorganic decomposition, respectively. Moreover, the coal/biomass blended fuels show the combined weight loss decomposition behaviors. Even though the incorporation of biomass wastes into lignite coal improved the ignition temperature of the blended fuels, the ignition and combustion index are worsened. This might be because biomass wastes, used in this present work, possess a lower values of maximum combustion rate and the less combustion reactivity in high temperature region, compared to lignite coal. From the kinetic studies, the average apparent activation energies of C75CR25, C75PKS25 and C75RH25 cocombustion calculated from KAS method are reported at $105.25,179.66$ and $121.84 \mathrm{~kJ} / \mathrm{mol}$, respectively. In order to maintain the combustion performance with regarding to the concerned environmental issues, it would be recommended that the co-combustion of coal/biomass, $75 / 25$ wt.\%/wt.\% in particular, can be utilized for energy production.

From the present experimental data, the outcome of this research would be useful for biomass waste utilization in terms of energy production and environmental concerns. In practical, the next step should be to evaluate all costs involved in the entire logistics chain, e.g., collecting, milling, storage, and transportation, in order to obtain the information for an economic point of view. It is also recommended that, moreover, the investigation of other biomass wastes as well as the solid products obtained from the biomass thermochemical conversion processes, e.g., torrefied biomass and biochar, should be accounted for coal/biomass waste co-combustion system.

\section{Acknowledgements}

The authors would like to thank Faculty of Science Energy and Environment, King Mongkut's University of Technology North Bangkok. This research was funded by King Mongkut's University of Technology North Bangkok. Contract no. KMUTNB-64-DRIVE-22.

\section{References}

1. S.G. Sahu, N. Chakraborty, P. Sarkar, Coal-biomass co-combustion: An overview, Renewable and Sustainable Energy Reviews, 39, (2014): 575-586

2. A. Hamisu, U.I. Gaya, Bi-template assisted sol-gel synthesis of photocatalytically-active mesoporous anatase $\mathrm{TiO}_{2}$ nanoparticles, Applied Science and Engineering Progress, Online First, (2021): 1-15

3. B. Ashok, M. Umamahesh, N. Hariram, S. Siengchin, A.V. Rajulu, Modification of waste leather trimming with in situ generated silver nanoparticles by one step method, Applied Science and Engineering Progress, 14, (2021): 236-246

4. M. Wzorek, R. Junga, E. Yilmaz, P. Niemiec, Combustion behavior and mechanical properties of pellets derived from blends of animal manure and lignocellulosic biomass, Journal of Environmental Management, 290, (2021): 112487

5. M.V. Gil, D. Casal, C. Pevida, J.J. Pis, F. Rubiera, Thermal behaviour and kinetics of coal/biomass blends during co-combustion, Bioresource Technology, 101, (2010): 5601-5608

6. Z. Liu, A. Quek, S. Kent Hoekman, M.P. Srinivasan, R. Balasubramanian, Thermogravimetric investigation of hydrochar-lignite co-combustion, Bioresource Technology, 123, (2012): 646-652

7. A. Toptas, Y. Yildirim, G. Duman, J. Yanik, Combustion behavior of different kinds of torrefied biomass and their blends with lignite, Bioresource Technology, 177, (2015): 328-336

8. F. Gyo, Z. Zhong, Optimization of the cocombustion of coal and composite biomass pellets, Journal of Cleaner Production, 185, (2018): 399-407

9. F. Guo, Y. He, A. Hassanpour, J. Gardy, Z. Zhong, Thermogravimetric analysis on the co-combustion of biomass pellets with lignite and bituminous coal, Energy, 197, (2020): 117147

10. S. Qi, Z. Wang, M. Costa, Y. He, K. Cen, Ignition and combustion of single pulverized biomass and coal particles in $\mathrm{N}_{2} / \mathrm{O}_{2}$ and $\mathrm{CO}_{2} / \mathrm{O}_{2}$ environments, Fuel, 283, (2021): 118956

11. S. Vyazovkin, A.K. Burnham, J.M. Criado, L.A. Perez-Maqueda, C. Popescu, N. Sbirrazzuoli, ICTAC kinetics committee recommendations for performing kinetic computations on thermal analysis data, Thermochimica Acta, 520, (2011): 1-19

12. P. Jongpradist, W. Homtragoon, R. Sukkarak, W. Kongkitkul, P. Jamsawang, Efficiency of rick husk ash as cementitious materials in high-strength cement-admixed clay, Advances in Civil Engineering, 2018, (2018): Article ID 8346319, 11 pages

13. G. Wang, J. Zhang, J. Shao, Z. Liu, G. Zhang, T. $\mathrm{Xu}$, J. Guo, H. Wang, R. Xu, H. Lin, Thermal behavior and kinetic analysis of co-combustion of waste biomass/low rank coal blends, Energy Conversion and Management, 124, (2016): 414-426

14. X.G. Li, Y. Lv, B.G. Ma, S.W. Jian, H.B. Tan, Thermogravimetric investigation on co-combustion characteristics of tobacco residue and high-ash anthracite coal, Bioresource Technology, 102, (2011): 9783-9787

15. A. Dashti, A.S. Noushabadi, M. Raji, A. Razmi, S. Ceylan, A.H. Mohammadi, Estimation of biomass higher heating value (HHV) based on the proximate analysis: Smart modelling and correlation, Fuel, 257, (2019): 115931 
16. D. Vamvuka, S. Sfakiotakis, Combustion behaviour of biomass fuels and their blends with lignite, Thermochimica Acta, 526, (2011): 192-199

17. J.-J. Lu, W.-H. Chen, Investigation on the ignition and burnout temperatures of bamboo and sugarcane bagasse by thermogravimetric analysis, Applied Energy, 160, (2015): 49-57

18. S.Y. Luo, B. Xiao, Z.Q. Hu, S.M. Liu, Y.W. Guan, Experimental study on oxygen-enriched combustion of biomass micro fuel, Energy, 34, (2009): 18801884

19. Y. Lin, Y. Liao, Z. Yu, S. Fang, X. Ma, The investigation of co-combustion of sewage sludge and oil shale using thermogravimetric analysis, Thermochimica Acta, 653, (2017): 71-78
20. K. Jayaraman, M.V. Kok, I. Gokalp, Combustion mechanism and model free kinetics of different origin coal samples: Thermal analysis approach, Energy, 204, (2020): 117905

21. C. Chen, S. Qin, F. Chen, Z. Lu, Z. Cheng, Cocombustion characteristics study of bagasse, coal and their blends by thermogravimetric analysis, Journal of the Energy Institute, 92, (2019): 364-369

22. K. Cong, F. Han, Y. Zhang, Q. Li, The investigation of co-combustion characteristics of tobacco stalk and low rank coal using a macro-TGA, Fuel, 237, (2019): 126-132

23. B. Mahanta, A. Saikia, U.N. Gupta, P. Saikia, B.K. Saikia, J. Jayaramudu, P.S. Sellamuthu, E.R. Sadiku, Study of low-rank high sulphur coal fine with biomass, Current Research in Green and Sustainable Chemistry, 3, (2020): 100023 